\title{
El currículo en la sociedad del conocimiento
}

\section{William R. Avendaño-Castro}

Universidad Francisco de Paula Santander, Cúcuta, Colombia wrac_ufps@hotmail.com

\section{Abad E. Parada-Trujillo}

Colegio Militar General Francisco de Paula Santander, Cúcuta, Colombia abadernesto@hotmail.com

\section{Resumen}

El objetivo de este estudio es analizar el concepto de currículo y sus relaciones con la llamada sociedad del conocimiento, la reproducción cultural y la cognición. Desde lo teórico se delimita el concepto de currículo enfatizando en su función principal: la reproducción de la cultura como base de la cognición de los individuos. Se analizan las implicaciones de la sociedad del conocimiento, asi como algunas de sus caracteristicas para encontrar puntos de conexión entre curriculo, cultura y sociedad de la información. Además, se exploran los principios y postulados de la teoría de la modificabilidad estructural cognitiva (MEC) de Reuven Feuerstein y el impacto de sus principios en la delimitación conceptual del currículo. Se concluye que la cultura de los sujetos es la base práctica para generar procesos cognitivos basados en el manejo de la información. La cultura y la sociedad del conocimiento ofrecen a la educación el contexto pertinente al cual se debe adaptar el currículo, a fin de responder a la sociedad actual y a los intereses de los educandos.

\section{Palabras clave}

Investigación sobre el curriculo, cultura, sociedad del conocimiento, educación. (Fuente: Tesauro de la Unesco).

Recepción: 2012-01-24 | Aceptación: 2012-12-05

Para citar este artículo / To reference this article / Para citar este artigo

Avedaño, W. R., Parada-Trujillo, A. E. (2013). El currículo en la sociedad del conocimiento cognitiva. Educ. Educ. Vol. 16, No. 1, pp. 159-174. 


\title{
The Curriculum in Knowledge Society
}

\begin{abstract}
This study analyzes the concept of a curriculum and its relationship to what is known as knowledge society, cultural reproduction and cognition. The theoretic concept of a curriculum is framed, and its main role underlined: to reproduce culture as the basis of cognition of individuals. The study analyzes the implications of a knowledge society as well as some of its characteristics in order to find a common ground between curriculum, culture and information society. Additionally, the study explores the principles and postulates of Reuven Feurenstein's theory of structural cognitive modifiability (SCM) and its impact on the principles of limiting the concept of curriculum. It concludes that a subject's culture is the practical ground on which to construct cognitive processes founded on information. Knowledge society must offer education the relevant context to which to adapt the curriculum in order to answer to today's society and respond to the needs of students.
\end{abstract}

Key Words

Research on curriculum, culture, knowledge society, education. (Source: Unesco Thesaurus).

\section{O currículo na sociedade do conhecimento}

\section{Resumo}

O objetivo deste estudo é analisar o conceito de currículo e suas relações com a chamada sociedade do conhecimento, com a reprodução cultural e a cognição. A partir da teoria, delimita-se o conceito de currículo e enfatiza-se sua função principal: a reprodução da cultura como base da cognição dos indivíduos. Analisam-se as implicações da sociedade do conhecimento, bem como algumas de suas características para encontrar pontos de conexão entre currículo, cultura e sociedade da informação. Além disso, exploram-se os princípios e postulados da teoria da Modificabilidade Estrutural Cognitiva $(M E C)$, de Reuven Feuerstein, e o impacto de seus princípios na delimitação conceitual do currículo. Conclui-se que a cultura dos sujeitos é a base prática para gerar processos cognitivos baseados na gestão da informação. A cultura e a sociedade do conhecimento oferecem à educação o contexto pertinente ao qual se deve adaptar o currículo a fim de responder à sociedade atual e aos interesses dos educandos.

\section{Palavras-chave}

Pesquisa sobre o currículo, cultura, sociedade do conhecimento, educação. (Fonte: Tesauro da Unesco). 


\section{Introducción}

El currículo es un término relativamente nuevo y ampliamente abordado por diferentes autores (Gvirtz y Palamidessi, 1998; Ianfrancesco, 2003), encontrándose en la literatura variedad de posiciones respecto de los temas propios del mismo. La aplicación del currículo desde el ámbito pedagógico ha generado un sin número de definiciones y ello ha conducido a que se transforme en una palabra polisémica. Sin embargo, es posible afirmar que el currículo "más que concebirse como lo que puede ser aprendido [...] [supone] un conjunto de experiencias mediante las cuales los que aprenden construyen una concepción del mundo más cercana a la concepción de los científicos" (Driver, 1988). De allí surge la idea de un currículo para la comprensión y la transformación del mundo, pues es innegable que la educación constituye un proceso de construcción de identidad y reproducción cultural. Es esencial hacer énfasis en esta concepción de la educación como objeto de estudio de la pedagogía pues es relevante para los procesos de formación redefinir esa imagen del sujeto dentro del contexto y de las relaciones que construye con los demás y con el medio en general.

La posición de Driver (1988) enfatiza en un enfoque constructivista de la pedagogía, por cuanto afirma que el objetivo del currículo es la búsqueda del saber epistémico, en otras palabras, la edificación del conocimiento por parte del mismo sujeto de aprendizaje. $Y$ no de cualquier saber sino de un conocimiento fortalecido y significativo. De alli que las instituciones educativas deban, de forma urgente, romper con el sistema escolar que ha mantenido las viejas formas dejando grandes vacíos en los niños, las niñas y los jóvenes que no encuentran una formación adecuada para desempeñarse en la sociedad globalizada (Pilonieta, 2010). A esto se refiere Monclús (2004) al afirmar que la "educación había caído [...] en esquemas, modelos, teorías y prácticas [que] venían repitiéndose desde hacía siglos de forma generalizada", y que a diferencia de la perspectiva de Monclús, la nuestra es que aún se siguen manteniendo, a pesar de observarse un pequeño cambio. También el currículo desde la posición del mediador es fundamental pues solo con él puede comprender el maestro su práctica pedagógica (lanfrancesco, 2003).

Estas viejas formas de educación se perciben aún en la transmisión del conocimiento de forma vertical y autoritaria, sin espacio a la crítica, y en espacios limitados (aulas de clase) con el fin de lograr la repetición del saber. Por ende, la transformación socioeconómica y cultural de la humanidad ha dejado atrás a la educación sin que esta llegue a comprender lo que implican todos estos hechos. Al respecto, Crozier (1998), citado por Monclús (2004), estima que "la idea desarrollada en Occidente en los años cincuenta y sesenta de que con la educación se podía reconstruir el mundo resulta más bien fracasada". Del mismo modo, con el currículo se ha presentado la misma situación pues Bobbit, quien hacia 1914 inició los estudios sobre este tema, expresó la idea de organizar la escuela conforme a una fábrica pues ello era lo que demandaba la sociedad. Se trataba de una educación enmarcada en la ingeniería y el utilitarismo (Gvirtz y Palamidessi, 1998). La educación, en este sentido, debía responder a ese tipo de sociedad, pero esta se transformó y los modelos educativos se quedaron suspendidos e inmutables.

Es necesario un sistema escolar dirigido hacia el desarrollo de procesos que contribuyan a la construcción de conocimientos sistematizados, abarcadores, significativos y con sentido, que respondan a las características de la sociedad del conocimiento, agitada por la crisis y por múltiples factores que, no queda de más mencionar, desconoce las instituciones educativas. Los inconvenientes y las dificultades que se suscitan en las escuelas se deben, en gran medida, a la formulación de currículos que no son pertinentes para los sujetos de aprendizaje y el medio sociocultural del cual hacen parte. En efecto, son escasos los currículos diseñados para mejorar los ni- 
veles de conocimiento, el manejo de la información, la potenciación de operaciones mentales y el desarrollo de las funciones cognitivas desde la óptica cultural de los sujetos de aprendizaje.

Por lo anterior, la Comisión de las Comunidades Europeas (2000) ha expresado que

la enseñanza como profesión se enfrenta a un cambio radical [...] los profesionales de la educación se convertirán en guías, tutores y mediadores. Su función consistirá en ayudar y apoyar a los que aprenden, que serán los que irán tomando las riendas de su aprendizaje,

y añade que

la tendencia es un aprendizaje a lo largo de la vida que debe acompañar la transición hacia una economía y una sociedad basadas en el conocimiento para que esta pueda tener éxito.

Por tanto, hablar de currículo es también abarcar los procesos de enseñanza-aprendizaje, las corrientes pedagógicas, la identidad cultural de las comunidades escolares, las políticas públicas educativas, las relaciones entre los sujetos que intervienen en el acto pedagógico, el conjunto de saberes necesarios para los individuos según su contexto, y el desarrollo de funciones cognitivas y operaciones mentales, todo desde el marco que configura la sociedad del conocimiento.

En este sentido, se busca responder a la relación que existe entre currículo con sociedad del conocimiento, reproducción cultural y campo cognitivo, pues resulta relevante pensar en una educación que no solo contribuya al manejo de la información sino que, además, constituya los pilares para la formación de niños, niñas y jóvenes con una identidad cultural y una posición política. Por esta razón, en el artículo se exponen los conceptos de currículo a fin de delimitar el mismo en un sentido epistémico, y con ello abordar las nociones de sociedad del conocimiento y modificabilidad estructural cognitiva (MEC) como teoría epistémica cuyos aportes al campo de la pedagogía y la educación han tenido un gran impacto en la actualidad.

\section{Método}

Para lograr el objetivo propuesto en este artículo se hace una revisión de fuentes teóricas contenidas en la amplia literatura académica que aborda el tema curricular, la sociedad del conocimiento y la educación como proceso de reproducción y transformación cultural. A fin de establecer las relaciones entre el currículo y el desarrollo cognitivo (desarrollo del pensamiento) se acude a los principios y las perspectivas asumidos por la teoría de la modificabilidad estructural cognitiva (MEC) de Reuven Feuerstein por cuanto su aplicación ha tenido gran auge alrededor del mundo. A partir de la reflexión de estos elementos se formula una propuesta epistémica que delimita la noción de currículo haciéndola coherente con las exigencias de la sociedad, las necesidades de los sujetos de aprendizaje y los avances en torno a la cognición.

\section{Evolución y actual perspectiva del currículo}

Para Hernández (1993), la concepción curricular menos elaborada es aquella en la que se considera el currículo como un plan de estudios. Ianfrancesco (2003) va más allá de esta perspectiva y a partir de un análisis de la literatura sobre el concepto de currículo permite inferir que dicho constructo es el resultado de un proceso evolutivo marcado por las características de la sociedad en cada momento histórico. Los autores citados por lanfrancesco (2003), entre los que se encuentran Sailor y Alexander (1954), Kearney y Cook (1960), Jhonson (1967), King (1976), Yung (1979), Berstein (1980), Schuber (1985), Apple (1986), Tormes (1992), entre otros, van desde considerar el currículo como una calificación de resultados escolares hasta percibirlo como el conjunto de "principios, propósitos y procesos de formación integral y social y medios para lograrla" (lanfrancesco, 2003). El desarrollo teórico del currículo como objeto de estudio tiene su nacimiento en los años cincuenta y va hasta el presente siglo con estudios que atacan las prácticas escolares actuales que se han quedado reducidas a modelos sociales de me- 
dio siglo atrás. Por ello es significativo analizar todo el proceso a fin de comprender a fondo la concepción actual de currículo.

Durante la década de los cincuenta aún no había un acuerdo conceptual sobre el término currículo, el cual podía ser observado desde la evaluación o desde una acción para disciplinar la escuela, asunto directamente relacionado con el paradigma comunicativo desarrollado en la posguerra para servir a los intereses políticos y bélicos que se gestaban con el orden mundial de las ideologías. Durante los años sesenta es identificable el criterio común de los autores para definir el currículo: guía. Ya en los años setenta son perceptibles otros elementos agregados por los autores a esta noción, entre los que se destacan la cultura, las experiencias, el conocimiento y lo social.

La década de los ochenta presenta una gran variedad de perspectivas teóricas sobre el currículo; estos autores retoman las concepciones de las décadas anteriores y formulan nuevas maneras de verlo. En este tiempo de contribución al currículo escolar resalta la visión pública del conocimiento, los valores y las creencias sociales. Ello es una respuesta a las agitadas dinámicas que precedieron la llegada de los años noventa en donde la humanidad observó un cambio drástico en el escenario de la geopolítica y en el panorama de la economía mundial.

En la década de los noventa la producción reflexiva y crítica alrededor del currículo incluye nuevos elementos asociados al término como la interdisciplinariedad, la cual es novedosa pues hay una tendencia hacia el desarrollo de procesos formativos integradores y abarcadores que permite la gestación de experiencias significativas y un mejor aprendizaje como lo explicara Morin (1999) en su documento Los siete saberes necesarios para la educación del futuro. Así, se deja a un lado la fragmentación del conocimiento en áreas y asignaturas en donde se asumía una postura descontextualizada y poco integradora del saber. De la misma forma, en este periodo se iniciaron los estudios sobre el currículo oculto com- prendido por los valores, las actitudes, los conocimientos y las destrezas que se gestaban en las escuelas sin definirse taxativamente. En efecto, este es el currículo que se ha mantenido para la formación de los niños, las niñas y los jóvenes, haciendo perdurar los antiguos modelos que respondían a la sociedad del siglo pasado.

Con la intención de romper con las viejas formas de reproducción escolar, en los años noventa, como lo explica lanfrancesco (2003), la gran cantidad de concepciones se resumen en la tendencia a considerar el currículo como los "principios, propósitos y procesos de formación integral y social y medios para lograrla". Esto no difiere de la idea desarrollada por otros autores como Posner (2005) quien en su análisis de las perspectivas curriculares de los últimos sesenta años encuentra que algunas responden: a) al modo en que se aprende y las formas en que se facilita dicho proceso; b) a los objetivos; c) a los contenidos y su organización; d) a la evaluación del proceso formativo; d) la relación real y la relación ideal entre escuela y sociedad.

En la recopilación que hace Gvirtz et al. (1998) se observan varias posturas sobre el currículo al igual que en Posner o lanfrancesco (Cuadro 1). Aquí se ven dos perspectivas de currículo que permiten enmarcar las tendencias en dos grandes grupos: el currículo como modelo de práctica y como proyecto práctico de elaboración colectiva.

Por su parte, Cox (2001) hace un acercamiento conceptual al currículo desde dos puntos de vista: el primero, refiriéndose al mismo como los "planes y programas de estudio, o el conjunto de contenidos, en un sentido amplio que, organizados en una determinada secuencia, el sistema escolar se compromete a comunicar"; y el segundo, describiendo el currículo como "una selección cultural con propósitos formativos, que organiza la trayectoria de alumnos y alumnas en el tiempo y que, en los contenidos, esquemas mentales, habilidades y valores que contribuye a comunicar, es un regulador mayor de su experiencia futura". 
Cuadro 1.

Perspectivas y tendencias del currículo

\begin{tabular}{|c|c|}
\hline Perspectiva & Tendencia \\
\hline \multirow{4}{*}{$\begin{array}{l}\text { Currículo como } \\
\text { modelo de } \\
\text { práctica }\end{array}$} & Cuerpo organizado de conocimientos. \\
\hline & Declaración de objetivos de aprendizaje. \\
\hline & Plan integral para la enseñanza. \\
\hline & $\begin{array}{l}\text { Conjunto de experiencias planificadas } \\
\text { para ayudar a los alumnos a conseguir los } \\
\text { objetivos según sus capacidades. }\end{array}$ \\
\hline \multirow{2}{*}{$\begin{array}{l}\text { Ampliado a } \\
\text { conjunto de } \\
\text { experiencias } \\
\text { significativas }\end{array}$} & $\begin{array}{l}\text { Oportunidades de aprendizaje que } \\
\text { proporciona la escuela. }\end{array}$ \\
\hline & $\begin{array}{l}\text { Experiencias de conocimiento y aprendizaje } \\
\text { en la escuela. }\end{array}$ \\
\hline \multirow{3}{*}{$\begin{array}{l}\text { Abordado como } \\
\text { compleja realidad } \\
\text { socializadora } \\
\text { (perspectiva } \\
\text { sociológica) }\end{array}$} & $\begin{array}{c}\text { Oportunidades y limitaciones que ofrece } \\
\text { la escuela adheridas a la administración } \\
\text { educativa y la enseñanza. }\end{array}$ \\
\hline & $\begin{array}{l}\text { También, el conjunto de influencias } \\
\text { formativas que la escuela ejerce } \\
\text { sistemáticamente, no explícitas y no } \\
\text { reconocidas formalmente (currículo oculto). }\end{array}$ \\
\hline & $\begin{array}{c}\text { Proceso social en donde hay cruce de } \\
\text { diversas prácticas. }\end{array}$ \\
\hline \multirow{3}{*}{$\begin{array}{l}\text { Currículo como } \\
\text { proyecto práctico } \\
\text { de elaboración } \\
\text { colectiva }\end{array}$} & $\begin{array}{l}\text { Puesta en práctica de una propuesta } \\
\text { educativa integrada por contenidos, } \\
\text { métodos, problemas y medios. }\end{array}$ \\
\hline & Proyecto integrado, global y flexible. \\
\hline & $\begin{array}{l}\text { Proporciona la base para planificar un curso } \\
\text { en cuanto al proyecto y al estudio empírico. }\end{array}$ \\
\hline
\end{tabular}

Fuente: autores a partir de la información de Gvirtz et al. (1998).

Conforme a la exposición de concepciones teóricas es posible identificar algunas de las características con las que debe contar el currículo actual, entre las que se encuentra la formación compleja, social, política y cultural; la contextualización de los procesos de formación; la flexibilidad; la coherencia con las políticas, y la visión social, entre otras. A continuación se exponen estas características las cuales permitirán una definición de currículo:

1. El currículo es un conjunto de procesos de formación porque implica la transversalidad de los saberes en situaciones concretas, además que busca un aprendizaje de tipo integrador que permita cambios relevantes y significativos en el sujeto que aprende. Con ello se aísla la idea del trabajo por áreas y asignaturas, la clase magistral (en torno al maestro) y el direccionamiento vertical de la enseñanza.

2. La formación es política y sociocultural porque la educación es ante todo un discurso y una acción de tipo social, cultural y político. Social en la medida que los procesos de formación tejen las relaciones humanas que conducen a la organización de las comunidades; cultural por cuanto incide en la reproducción de las conductas, las costumbres, la tradición y todo lo que implica este término (Coll, 1991); y política porque el ser humano actúa de forma dinámica dentro de una sociedad con el fin de incidir en ella, transformarla y determinarla, conforme a un acuerdo social consignado en la Constitución Política?.

3. Los procesos de formación están situados en el ámbito del educando y el mediador debido a que el currículo se centra en varios sujetos que están directa o indirectamente relacionados con los procesos de enseñanza y aprendizaje. Así, es imposible desconocer el medio de estos sujetos pues es alli, precisamente, en donde la formación recibida debe permitirle la construcción de mejores estados a fin de afrontar problemas de diversa indole ${ }^{2}$.

4. El currículo debe responder a una concepción del mundo y a unos propósitos identificados siendo necesario, por ende, un diseño previo, sin

1 La Constitución Política constituye un amplio referente para el currículo pues allí están consignados los principios, valores y fines de la sociedad colombiana, los derechos, las garantías y los deberes esenciales para alcanzar una convivencia sana y en procura de lograr la paz.

2 Ya nos hemos referido a la necesidad de un currículo basado en procesos de formación que tiendan a que el sujeto se enfrente con mejores capacidades a los problemas reales. Es preciso mencionar que "la resolución de problemas tiene una importancia cada vez más grande [y constituye] un objetivo para la educación en el nuevo milenio" (Gaulin, 2001). 
desconocer que por su flexibilidad puede ser modificado de acuerdo con circunstancias sobrevinientes o cambios en el contexto de los sujetos. Para Posner (2005) el currículo responde a un problema previamente identificado. Asi, en concordancia con lanfrancesco (2003), una de las tres funciones de la investigación curricular se resume en la identificación de problemas o puntos críticos. Por tanto, el currículo da respuesta opcional a una necesidad que la escuela considera debe ser satisfecha y que tiene relevancia en la formación de los sujetos de aprendizaje. Sin embargo, no solo se puede responder a unos puntos críticos locales pues son innegables los efectos de la globalización, la sociedad del conocimiento o de la información, los avances tecnológicos y las transformaciones en materia sociocultural, politica, económica y ambiental, entre otros tantos. De este modo, el currículo tiene un contexto amplio y uno reducido los cuales deben ser considerados en la construcción del mismo, sin excluirse el uno del otro.

5. El currículo es flexible por cuanto permite adaptarse a los cambios del entorno o de los sujetos, conforme a sus necesidades y los requerimientos propios de las circunstancias de vida en las que se ven inmersos. Un currículo rígido no permite el desarrollo de procesos de formación significativos y trascendentales. Como expresa Tedesco, "la producción de conocimientos requiere, además, un ambiente de creatividad y de libertad opuesto a toda tentativa autoritaria o burocrática de control del poder" (1998).

6. Es coherente con el direccionamiento estratégico de la escuela porque la misión, visión, los principios, los valores y las politicas generan un marco de acción y designan un destino colectivo de todos los que intervienen en el proceso. Es fundamental, en este sentido, que el currículo sea diseñado conforme a este direccionamiento en virtud de alcanzar la armonía y la coherencia de todo el sistema. Por ello expresa Hernández: "la creación o diseño curricular tiene que amarrar lo que la comunidad es y lo que pretende ser, teniendo en cuenta sus condiciones, su historia y las fuentes de su riqueza natural" (1993).

7. Responde a las necesidades de los sujetos partícipes pues la educación debe girar, como se mencionó, en torno a la creación de nuevos y mejores estados en los sujetos en el marco social, cultural y político. Se trata de una posición que resignifica la humanidad del hombre, fundamental en el mundo de hoy.

Si las anteriores características hacen parte del concepto currículo, es posible definirlo como el conjunto de procesos de formación politica y sociocultural contextualizados en el medio del educando y el mediador, previamente diseñados, flexibles y coherentes con el direccionamiento estratégico ${ }^{3}$ de la escuela y las necesidades de los sujetos partícipes en la práctica pedagógica. El currículo no puede estar sostenido sobre supuestos sino sobre certezas, necesidades y aspiraciones de seres humanos reales (Hernández, 1993), por lo que responde, inevitablemente, a una reproducción cultural de los sujetos de aprendizaje. Sumado a esta reflexión, el currículo debe considerar los avances en las disciplinas asociadas y que apoyan la investigación educativa. Por tanto, hablar de neurociencia, psicología, psicopedagogía, etc., es un asunto que le compete a la pedagogía, a la educación y, por ende, al currículo. Este último tendrá que considerar dentro de su diseño que:

...el ser humano se caracteriza por su alto nivel deflexibilidad [y que] las teorías que están apareciendo sobre el funcionamiento del sistema nervioso y de la fisiología del cerebro asi lo están confirmando, cuando nos hablan de plasticidad y flexibilidad del ser humano. Según esto, el individuo tiene el potencial oculto para aprender y puede modificarse a si mismo si lo desea (Ramirez y Roa, 2003).

3 El direccionamiento estratégico es entendido como un referente que incluye la misión, la visión, los principios, los valores y políticas de la institución educativa. 
Los avances en las ciencias que estudian la cognición son el resultado de considerar la aldea global como un gran espacio de producción y transformación de la información, en donde se gestan verdaderas comunidades de aprendizaje. Por ello, la sociedad del conocimiento es el tipo de sociedad a la cual deben responder las investigaciones curriculares y el diseño de los currículos actuales. A continuación se hace un análisis de las principales características de la sociedad del conocimiento, las cuales permitirán verificar si la delimitación epistémica realizada al concepto de currículo es apropiada a este modelo de sociedad imperante en pleno siglo XXI.

\section{La sociedad del conocimiento y currículo}

La sociedad del conocimiento ha venido para imponer toda clase de efectos sobre los seres humanos y a exigir de ellos una nueva perspectiva de la realidad, una moderna manera de ver los fenómenos desde los cambios permanentes que suscita (Herrera y Dridiksson, 1999), en otras palabras, a inferir en el campo cultural de los sujetos. Todo esto se debe a la reunión de varios factores que, asociados, generaron variaciones en el modus vivendi del hombre creando crisis por "las nuevas formas de organización social, económica y política" (Tedesco, 1998). Entre estos factores se encuentran la economía, el auge tecnológico y científico, la evolución en los medios de comunicación, una transformación de la conciencia humana y el manejo efectivo de la comunicación por las comunidades de aprendizaje. Así, Stiepovich (2004) encuentra que algunas de las características de la sociedad actual se resumen en: "la economía mundial, basada en conocimiento, la velocidad creciente de su recambio, la vinculación efectiva entre productores y consumidores de ese conocimiento, así como la articulación entre innovación, ciencia y tecnología".

En una forma simple, la sociedad de la información conduce a pensar en lo que el conocimiento representa en las comunidades actuales. La expresión sociedad del conocimiento: quiere decir, nada másy nada menos, queel saber y el conocimiento son los parámetros que gobiernan y condicionan la estructura y composición de la sociedad actual y son, también, las mercancías e instrumentos determinantes del bienestar y progreso de los pueblos"(Mateo, 2006).

Por ello, la sociedad se ha estructurado a través de comunidades que manejan la información, aprenden con ella, la procesan y la producen para ser transmitida, revalorada, estudiada, como un ciclo en permanente movimiento ${ }^{4}$. En este sentido también lo aclara Monroy (2005), que indica que "nuestras sociedades [...] son sociedades de la información, sociedades del aprendizaje, sociedades del conocimiento y sociedades educativas" que trabajan en red (García, 2001).

De cierta manera, Mateo (2006) nos demuestra con sus aportes que hay una sed del hombre por el conocimiento, la aprehensión y la producción del mismo, como si se tratase de un Renacimiento o de un Siglo de las Luces. Entonces, si la historia parece demostrar que ya el conocimiento ha jugado un rol definitivo y trascendental en la evolución de los pueblos, ¿qué diferencia a esta nueva ansia por la apropiación del conocimiento? La respuesta es muy sencilla, "la velocidad con que se genera y la rapidez con que se transmite" (Mateo, 2006) gracias a los medios de comunicación, la tecnología y el desarrollo de la ciencia imponiéndose a la economía basada en la productividad (Castells, 1997).

Por tanto, un elemento que debe ser incorporado al currículo es el uso de los medios y las tecnologías de la información a fin de afianzar competencias que le permitan a los sujetos comunicar de manera efectiva, en otras palabras, saber decir y saber interpretar la información que tienen a su alcance. Y con este enfoque que se genera la investigación, la creatividad y la innovación juegan un rol importante. En efecto, el conocimiento tiene como bases la investigación, el desarrollo y la innovación

4 El conocimiento es infinitamente susceptible de ampliación y su uso no lo desgasta, por el contrario, lo ensancha (Tedesco, 1998) 
por un lado, y por otro la enseñanza (Mateo, 2006), de aquí se desprende la conexión entre sociedad del conocimiento y educación, pues esta última debe brindar los saberes preestablecidos para impulsar la investigación, el desarrollo y la innovación, proceso que potencie la mejora de los conocimientos y la creación de nuevos. Así, la enseñanza es un factor clave y crítico en el desarrollo de la sociedad toda vez que debe formar a los futuros ciudadanos y científicos (Driver, 1988) que se enfrentarán a los problemas de la realidad con una comprensión de estos "a partir de perspectivas tanto actuales como históricas" (Garret, 1987). Por ende, es fundamental que la educación sea asumida como un asunto de seguridad nacional o una política de Estado (Pilonieta, 2010) para el bienestar de los pueblos, en donde el manejo y la producción del conocimiento constituyan aspectos vitales del currículo.

En esta medida la sociedad del conocimiento, con todos sus efectos 5 , incide en la educación (Ginés, 2004) y, por ende, en el currículo. Este tipo de sociedad obliga a las instituciones a proyectarse conforme al medio, ese contexto global que tampoco es independiente al de cada uno de los sujetos:

Vivimos en una economía del conocimiento, en una sociedad del conocimiento. Las economías del conocimiento son estimuladas y dirigidas por la creatividad y la inventiva. Las escuelas de la sociedad del conocimiento tienen que crear estas cualidades, si no su gente y sus naciones se quedarán atrás (Hargraves, 2003).

La enseñanza y el aprendizaje se concretan en el currículo, y si los hombres se encuentran inmersos en una sociedad basada en el conocimiento, entonces el objetivo del currículo es la formación de sujetos sociales y políticos en un medio cultural caracterizado por la gestión del conocimiento y el manejo permanente de un gran cúmulo de información. Para ello, la selección de la información que se busca transmitir debe ser valorada por el me-

5 Los cuales implican grandes transformaciones "no planificadas que [...] afectan la forma como nos organizamos, como trabajamos, como nos relacionamos y como aprendemos" (García, 2001). diador conforme a los intereses y el contexto de los educandos, de una manera crítica a fin de mejorar su calidad de vida. Por esta razón, el mediador debe incluir el uso de medios y tecnologías que desplacen los cuadernos y los tableros, y que permitan el desarrollo de experiencias más ligadas a la sociedad del conocimiento y a la integración de los saberes. Esta es la coherencia a la cual se debe llegar: contexto del sujeto de aprendizaje, direccionamiento estratégico de la institución y formación concreta en el aula.

Si los anteriores elementos hacen parte del actual currículo, lo es también el modelo pedagógico definido por los aportes de las teorías ofrecidas por la psicología y la psicopedagogía desarrolladas en torno al tema enseñanza-aprendizaje. Esto significa que la relación entre mediador y sujeto de aprendizaje es el medio que posibilita que el currículo se concrete efectivamente en los ambientes de aprendizaje. Al tenor de esta idea, la modificabilidad estructural cognitiva (MEC) y el modelo de la experiencia de aprendizaje mediado (EAM) resultan ser alternativas de gran impacto para el desarrollo de un currículo basado en la sociedad del conocimiento y el manejo de la información.

\section{La teoría de la modificabilidad estructural cognitiva (MEC)}

La modificabilidad estructural cognitiva es una teoría creada tras varias décadas de investigación del doctor Reueven Feuerstein. Su origen se remonta a las secuelas de la Segunda Guerra Mundial y al trabajo con la población judía sobreviviente del holocausto nazi. Su principio es abarcador y hace parte de las pedagogías de la esperanza no solo por considerar al hombre como un ser que puede ser modificado pese a cualquiera de las circunstancias en que se encuentra y a la deprivación cultural ${ }^{6}$ en que se halla sino que, además, sus descubrimientos nacen de "la adaptación de los niños judíos procedentes de

$6 \quad$ La deprivación cultural es definida por Feuerstein como la incapacidad de los sujetos para responder adecuadamente a los estímulos. 
otros países, portadores de su cultura propia y por consiguiente, costumbres diferentes" (Orrú, 2003) al sistema escolar en Israel.

En la génesis de la teoría es notoria la relación entre la cultura de los sujetos y los procesos de enseñanza y aprendizaje de tipo cognitivo. Desde la concepción de Feuerstein la mediación del maestro y de la madre le facilita al niño, la niña y el joven el acceso al mundo cultural, histórico, científico, social y moral (Velarde, 2008). Por ello, afirma Feuerstein (1970) que la MEC tiene un enfoque de modificación activa dirigida hacia el cambio del individuo y su adaptación al medio.

Abordando los inicios de las investigaciones de Feuerstein, Velarde (2008) describe que el trabajo de investigación empezó con el intento de "modificar los niveles cognitivos de esos niños, muchos de los cuales contaban ya con 14 años de edad y presentaban severas resistencias para adquirir aprendizajes complejos como la lectura"; todo este trabajo ha conducido a tomar una nueva postura sobre la estimulación de la inteligencia, la cual es un compromiso democrático pues ello implica que todo ser humano puede ser modificado en sus estructuras a fin de conducirlo a nuevos estados más dignos y al desempeño de un papel activo dentro de la sociedad. Se trata, en sintesis, de una responsabilidad social de todas las comunidades.

La teoría de la modificabilidad estructural cognitiva a pesar de haber tenido un origen especial, en un espacio y tiempo específico, ha sido aplicada a miles de niños, niñas y jóvenes con diferentes problemas cognitivos y en todos los niveles en más de 70 países en todo el mundo. Los resultados no solo han sido validados por Feuerstein y el instituto que lidera a nivel mundial sino por otros estudios realizados, en donde se demuestra un mejoramiento en las funciones cognitivas de los sujetos intervenidos y en el rendimiento escolar de los mismos (García y Porras, 2000; Himmel et al., 1996; Velarde, 2008; Varela, Gramacho y Melo, 2006; Cerrillo, 2001). El auge de la teoría de la modificabilidad estructural cognitiva representa una oportunidad para muchas personas limitadas por el sistema y esto se debe a que la perspectiva de Feuerstein se basa en el convencimiento de que el "organismo humano tiene la capacidad única de llegar a modificarse en una variedad de funciones cognitivas y motivacionales, adaptándose a los cambios y respondiendo a las demandas y situaciones nuevas" (Himmel et al., 1996). En este sentido Feuerstein, al igual que otros, considera el organismo humano como un sistema abierto al cambio y a la modificación (Prieto, 1989), lo cual solo es posible si se considera la inteligencia como un proceso dinámico excluyendo la vieja idea positivista de entenderla como algo fijo e inmutable. Siendo así, es posible modificar al sujeto para que este responda adecuadamente a los estímulos del medio, y es la experiencia de aprendizaje mediado la que facilita este proceso.

La mediación o experiencia de aprendizaje mediado (EAM) es el principal instrumento del maestro en el desarrollo de los procesos de formación desde la perspectiva de la modificabilidad estructural cognitiva. Ella responde a una de las formas en que el sujeto puede interactuar con el medio. Describe Ramírez et al. (2003) que:

El ser humano, desde que nace, interactúa con su medio de dos formas distintas: por un lado, el acercamiento a su entorno puede realizarse autónomamente, sin intermediarios. En este caso, el sujeto se expone directamente a los estímulos y elabora una respuesta en función de estos; por otro lado, la persona humana puede encontrarse con un adulto (padre, madre, maestro, etc.) que actúe como mediador entre el organismo y el entorno.

La mediación conduce al uso efectivo del medio y al suministro de los estímulos adecuados a los niños, las niñas y los jóvenes para producir los efectos esperados. Hay una relación estrecha entre lo que plantea Feuerstein con la mediación y los 
postulados de Vygostky (1985) respecto de la zona de desarrollo próximo definida como:

... la distancia entre el nivel real de desarrollo, determinado por la capacidad de resolver independientemente un problema, y el nivel de desarrollo potencial, determinado a través de la resolución de un problema bajo la guía de un adulto o en colaboración con un compañero más capaz. El estado del desarrollo mental de un niño puede determinarse únicamente si se lleva a cabo una clasificación de sus dos niveles: del nivel real del desarrollo y de la zona de desarrollo potencial (Vygotsky, 1985).

Por tanto, la zona de desarrollo próximo elaborada por la teoría de Vygostky (1985) constituye un aspecto relevante para la teoría de Feuerstein en lo que respecta a la mediación, pues esta corresponde al nivel de desarrollo potencial del sujeto, es decir, a la acción del mediador sobre el organismo y los estímulos. Para que dichos estímulos logren ser capturados por el sujeto de una forma adecuada se requiere que las funciones cognitivas se encuentren plenamente desarrolladas.

Las funciones cognitivas para Feuerstein et al. (1994) son prerrequisitos de la inteligencia para que la información proveniente de las fuentes de estímulos sea tratada de forma adecuada y el organismo alcance la autorregulación. Dicha autorregulación tiene una especial relación con la adaptación, también abordada por Piaget (2005) en sus estudios cuando afirma que el hombre en el proceso de aprendizaje se modifica (acomodación), lo que presupone una adaptación que se asocia al sentimiento de satisfacción del sujeto. Feuerstein et al. (1994) dividen las funciones cognitivas de acuerdo con su campo de acción en el pensamiento: input, elaboration, output (entrada, elaboración y salida). En la fase de entrada está la percepción clara y precisa, el comportamiento planificado, la correcta orientación espacial, el uso de instrumentos verbales, entre otras. En la fase de elaboración están las funciones asocia- das a la percepción de un problema y su definición, la capacidad para distinguir datos relevantes de los irrelevantes, amplitud del campo mental, construcción de relaciones, etc. La fase de salida del pensamiento incluye la funciones cognitivas relacionadas con la comunicación no egocéntrica, respuestas sistematizadas, instrumentos verbales adecuados, conducta sistematizada y planificada, entre otras.

Las funciones cognitivas permiten la correcta entrada y salida de información de la mente del sujeto, y el manejo de esta información le corresponde a las operaciones mentales. Las operaciones mentales son definidas como las acciones interiorizadas a nivel de pensamiento que realiza un sujeto con la finalidad de manejar la información y que no pueden operar correctamente cuando existen deficiencias cognitivas. Menciona Prieto (1989) citando a Feuerstein (1980) que "son un conjunto de acciones interiorizadas, organizadas y coordinadas, en función de las cuales realizamos la elaboración de la información que recibimos de las fuentes externas e internas".

Las operaciones mentales constituyen acciones específicas sobre la información y estas, desde la teoría de la modificabilidad estructural cognitiva, van desde la manipulación y la acción, pasando por la organización perceptiva y el manejo de las imágenes hasta el dominio del aparato simbólico (Bruner, 2004). Esto sugiere un proceso de formación basado en el manejo de la información cuyo desarrollo se caracteriza por niveles cada vez mayores de complejidad y abstracción. Las operaciones mentales en orden de menor a mayor nivel de abstracción son la identificación, la diferenciación, la representación mental, la transformación mental, la comparación, la clasificación, la codificación, la decodificación, la proyección de relaciones virtuales, el análisis, la síntesis, la inferencia lógica, el razonamiento analógico, el razonamiento hipotético, el razonamiento transitivo, el razonamiento silogístico y el pensamiento divergente (Pilonieta, 2010). 
Las acciones interiorizadas (operaciones mentales) del sujeto con las que aborda la información y da tratamiento a la misma, no están desligadas de la cultura de los hombres. Para Bruner (2004) la cognición —es decir, el aprendizaje y el manejo de la información- tiene como factores esenciales los valores sociales y el lenguaje. En este sentido, el desarrollo cognitivo de los sujetos está marcado por la cultura pues es en este campo en donde la información adquiere un significado para el sujeto desde su propia realidad. Por ello el hombre es considerado como un "individuo que vive, piensa y actúa en el marco de su cultura y de la premisa que la base de la cognición se encuentra en la praxis social" (Radford, 2006).

Es posible admitir que los presupuestos y principios de la teoría de la modificabilidad estructural cognitiva permitan el desarrollo de un currículo enfocado en la formación de sujetos pertinentes y coherentes con la sociedad del conocimiento pues dentro de las ideas que plantea se encuentra la de desarrollar elementos básicos para un adecuado manejo de la información a nivel de pensamiento. En efecto, este es uno de los mayores problemas en las escuelas y la ceguera producida por los modelos pasados no permite a los maestros en la actualidad encontrar las verdaderas causas de los bajos niveles de aprendizaje de muchos niños, niñas y jóvenes con deprivación cultural. De la misma forma, como se admite en la teoría, la cultura de los sujetos de aprendizaje es un importante factor que determina los procesos de enseñanza y aprendizaje. Por este motivo Feuerstein et al. (1994) incluyen como elemento que incide en la mediación del maestro los factores etiológicos distales de carácter exógeno, es decir, los estímulos ambientales, el estatus socioeconómico, el nivel educativo y la diferencia cultural.

La modificabilidad estructural cognitiva es una teoría que constituye todo un sistema de asumir la enseñanza y el aprendizaje, el rol de los sujetos que intervienen en el acto pedagógico y una manera de vivir la escuela. Se trata de una teoría que le apuesta a los cambios trascendentales y permanentes en los individuos pese a cualquier condición que lo limite pues, como se mencionó, el organismo humano se considera como un sistema abierto, además que "los determinantes distales (factores genéticos, herencia, maduración, factores orgánicos, emociones, estímulos ambientales, estatus socioeconómico, nivel educativo, diferencia cultural) no producen deterioro irreversible en los sujetos" (Prieto, 1989).

Feuerstein (1980) expresa que "cuando queremos producir cambios en el individuo, no estamos queriendo que el cambio producido permanezca limitado a la cosa aprendida apenas". Por tanto, lo que se busca con la implementación de un currículo basado en los aportes de Feuerstein es que los sujetos puedan realizar observaciones, plantear preguntas, examinar fuentes de información, planificar investigaciones, analizar los resultados de la experiencia, manipular datos (recoger, analizar e interpretar), plantear preguntas, explicaciones y predicciones, y comunicar resultados (Jiménez, 1998).

Para la formulación del currículo se puede atender a la ruta que dispone Taba (1974), reiterando que es viable y positivo enmarcarse en la teoría de la MEC. La autora señala los pasos para el diseño curricular en las siguientes etapas: reflexión y definición de los objetivos de la educación?, diagnóstico, selección de experiencias, organización del contenido y del aprendizaje, evaluación de los resultados y definición del modelo de enseñanza y aprendizaje. Estos elementos se deberán establecer bajo los criterios de la mediación con el fin de articularlos (secuencia, continuidad e integración). Es preciso señalar, para la comprensión plena de lo que es la articulación en el diseño curricular, que en el currículo "se concretan y toman cuerpo una serie de principios de índole diversa —ideológicos, pedagógicos, psicopedagógicos-, que tomados en su conjunto muestran la orientación general del sistema educativo" (Coll, 1991).

$7 \quad$ Es muy común en los maestros que frente a los objetivos que se persiguen dentro de las clases exista un vacío y poca claridad y coherencia (Tyler, 1986) con el resto del sistema, situación que obstruye el papel del currículo dentro de las escuelas. 
La perspectiva sobre desarrollo humano elaborada por la teoría de la modificabilidad estructural cognitiva en la cual se considera al hombre como un organismo abierto al cambio siempre y cuando exista una acción mediadora es adaptable a la noción de currículo propuesta en el trabajo toda vez que este supone un proceso de formación política y sociocultural contextualizado en un medio que, como ya se ha explicado, corresponde al de la sociedad del conocimiento caracterizada por el cambio permanente, la crisis y las transformaciones profundas. Así, reconocer que el hombre es un sujeto abierto al cambio supone también una adaptación constante a ese medio llamado sociedad del conocimiento, para lo cual la teoría de la modificabilidad estructural cognitiva ha elaborado la experiencia de aprendizaje mediado como dispositivo que activa los procesos de enseñanza y aprendizaje basados en el manejo de la información y la transmisión cultural.

\section{Conclusiones}

La sociedad del conocimiento exige de los procesos de formación y, por ende, del currículo, adaptaciones bajo el principio del conocimiento y la información como valores esenciales en los ciudadanos modernos ya que "las sociedades actuales están directamente relacionadas con el nivel de formación de sus ciudadanos y de la capacidad de innovación y emprendimiento que estos posean" (García, 2001). Por tanto, el currículo deberá procurar un verdadero cambio dentro de la conducta humana entendida esta como las modificaciones tanto en el pensamiento como en el sentimiento y las acciones manifiestas (Tyler, 1986), objetivos que son asumidos en la teoría de la modificabilidad estructural cognitiva.

El recorrido realizado por diferentes tópicos teóricos ha dejado sobre la mesa varias posiciones que deben seguir siendo retomadas por la investigación curricular en virtud de avanzar en la construcción de su objeto de estudio. Es importante destacar cómo la neurociencia y sus resultados acerca del funcionamiento del cerebro y la mente han impactado la educación y han validado los postulados de la teoría de la modificabilidad estructural cognitiva. En este sentido, las personas involucradas en la elaboración del currículo no pueden desconocer estos aportes y las últimas tendencias.

Así las cosas, la definición propuesta en este documento acerca de currículo debe ser ampliada en el siguiente sentido: currículo es el conjunto de procesos de formación en el campo cognitivo (funciones cognitivas y operaciones mentales) con base en el sistema sociocultural y político que contextualiza el medio del educando y el mediador, previamente diseñados, flexibles y coherentes con el direccionamiento estratégico de la escuela y las necesidades de los sujetos partícipes en la práctica pedagógica con el fin de disminuir la deprivación cultural.

El trabajo de aquí en adelante puede estar sustentado en definir cuál debe ser el diseño curricular pertinente e idóneo basado en los principios, postulados y perspectivas de la modificabilidad estructural cognitiva. Para ello se sugiere abordar —y modificar, de ser necesario (adaptarlo) — el mapa cognitivo de Feuerstein, el cual permite hacer una revisión no solo de los contenidos sino, además, de las funciones cognitivas, las operaciones mentales, la modalidad de intervención, las fases de pensamiento implicadas y los niveles de complejidad, de abstracción y de eficiencia que cada estudiante asume frente a situaciones específicas, lo que permitiría que los sujetos de aprendizaje puedan verse enfrentados a diversos problemas ${ }^{8}$ mediante "la activación de conocimientos factuales y conceptuales específicos, así como el dominio de técnicas y estrategias que en muchos casos difieren de un área a otra" (Pozo et al., 1994) pero que de igual forma responden a la sociedad del conocimiento.

8 La búsqueda de procesos de formación basados en la mejora de la competitividad del sujeto para afrontar problemas es aplicable tanto a los currículos de las escuelas como de las universidades (Herrera y Didriksson, 1999). 
ISSN $0123-1294$ | Educ.Educ. Vol. 16. No. 1 | Enero-abril de 2013 | pp. 159-174.

Universidad de La Sabana | Facultad de Educación

\section{Referencias}

Bruner, J. S. (2004). Desarrollo cognitivo y educación. Madrid: Morata.

Castells, M. (1997). La era de la información (Vol1): Economía, sociedad y cultura: La Sociedad Red. Madrid: Alianza.

Cerrillo Martín, M. (2001). Atención a la diversidad por medio de un programa de intervención socio-cognitiva. Tendencias Pedagógicas (6), 187-199.

Coll, C. (1991). Psicología y currículum. Barcelona: Paidos.

Cox, C. (2001). El currículum escolar del futuro. Revista Perspectivas, 4 (2), 213-232.

Comisión de las Comunidades Europeas (2000). Documento de trabajo de los servicios de la comisión: Memorándum sobre el aprendizaje permanente. Bruselas: SEC.

Driver, R. (1988). Un enfoque constructivista para el desarrollo del currículo en ciencias. Enseñanza de las Ciencias, 6 (2), 109-120.

Feuerstein, R. (1970). A dinamic approach to the causation, prevention, and alleviation of retarted performance. En Haywood, H. C. (ed.). Socio-cultural Aspects of mental retardation (pp. 341-377). New York: AppletonCentury-Crofts.

Feuerstein, R. (1980). Instrumental Enrichment: An intervention program for cognitive modifiability. Baltimore: University Park Press.

Feuerstein, R., Klein, P. y Tannenbaum, A. (1994). Mediated Learning Experience (MLE): theoretical, psychosocial and learning implications. 2 ed. Tel Aviv: Freund Publishing House Ltd.

García, C. M. (2001). Aprender a enseñar para la sociedad del conocimiento. Revista Complutense de Educación, $12(2), 531-593$.

García Romero, I. y Porras Vallejo, R. (2000). Condiciones para la aplicación de la adaptación del Programa de Enriquecimiento Instrumental (PEI) de Feuerstein en el primer ciclo de educación primaria. Kikirikí [Revista electrónica] (41). Recuperado el 14 de octubre de 2011 de: http://www.quadernsdigitals.net/index. php?accionMenu=hemeroteca.VisualizaArticulolU.visualiza\&articulo_id=1169

Garret, R. M. (1987). Resolución de problemas y creatividad: implicaciones para el currículo de ciencias. Enseñanza de las Ciencias, 6 (3), 224-230.

Gaulin, D. C. (2001). Tendencias actuales de la resolución de problemas. Sigma (19), 51-63.

Ginés Mora, J. (2004). La necesidad del cambio educativo para la sociedad del conocimiento. Revista Iberoamericana de Educación (035), 13-37. 
Gvirtz, S. y Palamidessi, M. (1998). El ABC de la tarea docente: curriculum y enseñanza. Argentina: Aique.

Hargraves, A. (2003). Enseñar en la sociedad del conocimiento (La educación en la era de la inventiva). Barcelona: Octaedro.

Hernández, D. (1993). Enfoques curriculares para el siglo XXI. Revista de Educación y Cultura, 39.

Herrera, A. y Didriksson, A. (1999). La construcción curricular: innovación, flexibilidad y competencias. Educación superior y sociedad, $10(2), 29-52$.

Himmel, E., Álvarez-Salamanca, E., Díaz, C., Fuentes, S. y Villalón, M. (1996). Funciones cognitivas y habilidades instrumentales necesarias para los aprendizajes formales en el niño. International Journal of Early Childhood, 28 (2), 15-20.

lanfrancesco, G. M. (2003). Nuevos fundamentos para la transformación curricular - a propósito de los estándares. Bogotá: Magisterio.

Jiménez, A. (1998). Diseño curricular: Indagación y razonamiento con el lenguaje de las ciencias. Enseñanza de las Ciencias, 16 (2), 203-216.

Mateo, J. L. (2006). Sociedad del conocimiento. Ciencia, Pensamiento y Cultura Arbor, CLXXXII (718), 145-151.

Monclús, A. (2004). Educación y cruce de culturas. México: Fondo de Cultura Económica.

Monroy, B. (2005). Pedagogía cognitiva en las sociedades de la información. Documento de trabajo (inédito). Bogotá: Universidad Externado de Colombia.

Morin, E. (1999). Los siete saberes necesarios para la educación del futuro. París: Unesco.

Orrú, S. E. (2003). Reuven Feuerstein y la teoría de la Modificabilidad Estructural Cognitiva. Revista de Educación, $332,33-54$.

Piaget, J. (2005). Inteligencia y afectividad. Buenos Aires: Aique Grupo Editor.

Pilonieta, G. (2010). Modificabilidad Estructural Cognitiva y Educación. Bogotá: Magisterio.

Posner, G. (2005). Análisis del currículo. México: McGraw Hill.

Pozo, J. I., Del Puy Pérez, M., Domínguez, J., Ángel Gómez, M. y Postigo, Y. (1994). La solución de problemas. Madrid: Santillana.

Prieto Sánchez, M. D. (1989). Modificabilidad cognitiva y PEI. Madrid: Bruño.

Radford, L. (2006). Semiótica cultural y cognición. En Cantoral Uriza, R., Covián Chávez, O., Farfán, R. M., Lezama Andalón, J. y Romo Vázquez, A. (eds.). Investigaciones sobre Enseñanza y aprendizaje de las matemáticas. Un reporte iberoamericano (pp. 669-689). México: Díaz de Santos. 
ISSN 0123-1294 | Educ.Educ. Vol. 16. No. 1 | Enero-abril de 2013 | pp. 159-174.

Universidad de La Sabana | Facultad de Educación

Ramírez Fernández, S. y Roa Venegas, J. M. (2003). El programa de enriquecimiento instrumental de Feuerstein: una aproximación teórica. Eúphoros, 6, 261-270.

Stiepovich Bertoni, J. (2004). Sociedad y Conocimiento. Ciencia y Enfermería, 10 (2), 7-7.

Taba, H. (1974). Elaboración del currículo. Buenos Aires: Troquel.

Tedesco, J. C. (1998). Educación y sociedad del conocimiento y de la información. Revista Colombia de Educación (36/37), 139-154.

Tyler, R. W. (1986). Principios básicos del currículo. Buenos Aires: Troquel.

Varela, A., Gramacho, A. y Melo, C. (2006). Programa de Enriquecimiento Instrumental (PEI): alternativa pedagógica que responde al desafío de calidad en educación. Diversitas - Perspectivas en psicología, 2 (2), 297-310.

Velarde Consoli, E. (2008). La teoría de la modificabilidad estructural cognitiva de Reuven Feuerstein. Investigación Educativa, 12 (22), 203-221

Vygotsky, L. S. (1985). Pensamiento y lenguaje. Pléyade: Buenos Aires. 